\title{
Pooled Results of VP-102 Safety and Efficacy in Phase 3 Trials for Molluscum Contagiosum by Fitzpatrick Skin Type (FST)
}

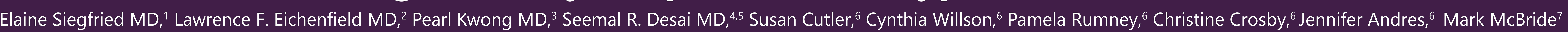

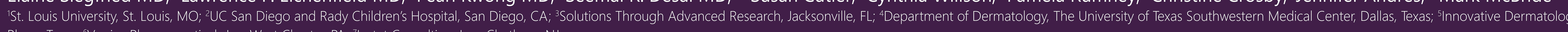

INTRODUCTION

VP-102, a single-use proprietary drug-device combination product (cantharidin $0.7 \%$ ), is under investigation for treatment of molluscum contagiosum (MC). In 2 phase 3 trials, 528 subjects $\geq 2$ years with MC, were randomized (3:2) to receive topical application of VP-102 or vehicle.

- While most subjects were Caucasian VP-102:vehicle $(\mathrm{N}=277: 202$ [89.4\%:92.7\%]), there was representation across all FSTs. FST is a tool which assesses skin burn propensity during phototherapy but can be misused to imply constitutive skin color/ethnicity.

- Skin of Color patients with molluscum often experience pigmentary changes with or without treatment. However,

This post hoc analysis was designed to assess VP-102 efficacy (subjects achieving complete clearance (CC) (\%)) and safety by FST group, compared to the overall study population.

METHODS

CAMP 1 (NN=266) Two Randomized, Vehicle-Controlled, Double-Blind Phase 3 Trials \&

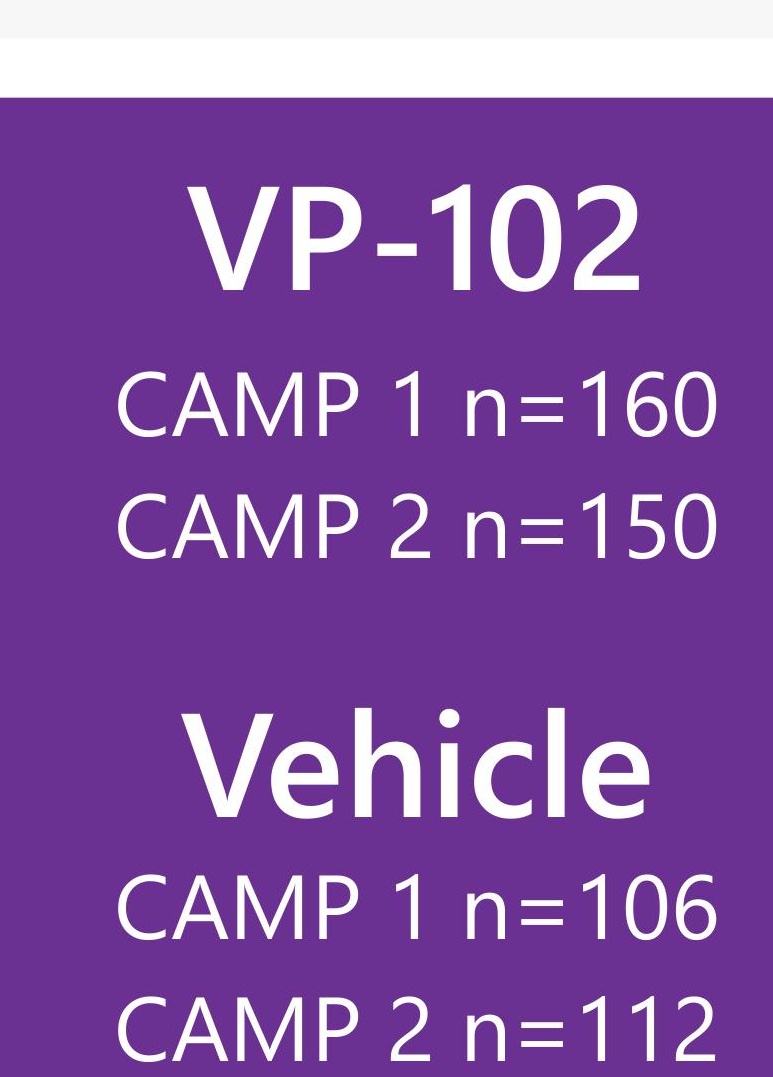

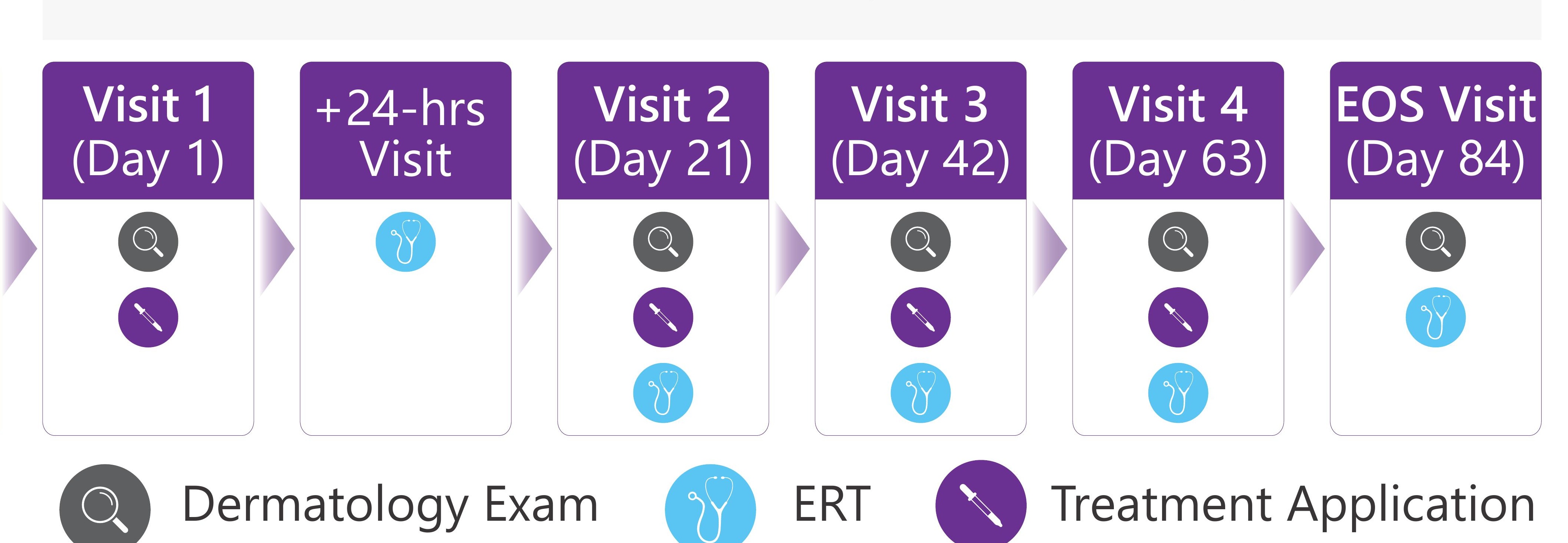

Q Dermatology Exam

- VP-102 or vehicle was applied to all baseline and new lesions once every 21 days until CC, or up to 4 applications.

- Subjects were evaluated at Days $21,42,63,84$.

- Treatment emergent adverse events (TEAEs) were assessed throughout the study.
DEMOGRAPHICS \& MEDICAL HISTORIES

\section{Baseline Characteristics}

\begin{tabular}{l|c|c|} 
& $\begin{array}{c}\text { VP-102 } \\
(\mathrm{n}=311)\end{array}$ & $\begin{array}{c}\text { Vehicle } \\
(\mathrm{n}=216)\end{array}$ \\
\hline $\begin{array}{l}\text { Age (years) } \\
\text { Mean (SD) }\end{array}$ & $7.5(6.7)$ & $6.8(5.8)$ \\
\hline $\begin{array}{l}\text { Median (Range) } \\
\text { Gender - no. (\%) } \\
\quad \text { Male }\end{array}$ & $6.0(2-60)$ & $6.0(2-54)$ \\
\hline $\begin{array}{l}\text { Race or Ethnic Group - no. (\%) } \\
\text { White }\end{array}$ & $156(50.2)$ & $111(51.4)$ \\
\hline Black or African American & $277(89.1)$ & $201(93.1)$ \\
\hline Asian & $14(4.5)$ & $7(3.2)$ \\
\hline $\begin{array}{l}\text { American Indian/Alaskan Native } \\
\text { Other }\end{array}$ & $6(1.9)$ & $1(0.5)$ \\
\hline
\end{tabular}

\section{Molluscum Medical Histories}

\begin{tabular}{|c|c|c|}
\hline & $\begin{array}{l}\text { VP-102 } \\
(\mathrm{n}=311)\end{array}$ & $\begin{array}{l}\text { Vehicle } \\
(n=216)\end{array}$ \\
\hline \multicolumn{3}{|l|}{ Baseline Lesion Count } \\
\hline Mean (SD) & $20.4(23.0)$ & $22.6(22.3)$ \\
\hline Median (Range) & $12.0(1-184)$ & $16.0(1-110)$ \\
\hline \multicolumn{3}{|c|}{ Atopic Dermatitis (AD) - no. (\%) } \\
\hline History or Active $A D$ & 50 (16.1) & 35 (16.2) \\
\hline Active $A D^{*}$ & $23(7.4)$ & $20(9.3)$ \\
\hline \multicolumn{3}{|c|}{ Fitzpatrick Skin Type - No. (\%) } \\
\hline 1 & $20(6.5)$ & $8(3.7)$ \\
\hline II & $81(26.1)$ & $71(32.6)$ \\
\hline III & $97(31.3)$ & $70(32.1)$ \\
\hline IV & $69(22.3)$ & $34(15.6)$ \\
\hline $\mathrm{v}$ & $33(10.6)$ & $32(14.7)$ \\
\hline VI & $9(2.9)$ & $3(1.4)$ \\
\hline
\end{tabular}

RESULTS

Complete Lesion Clearance by Fitzpatrick Skin Type (Pooled IIT Population)

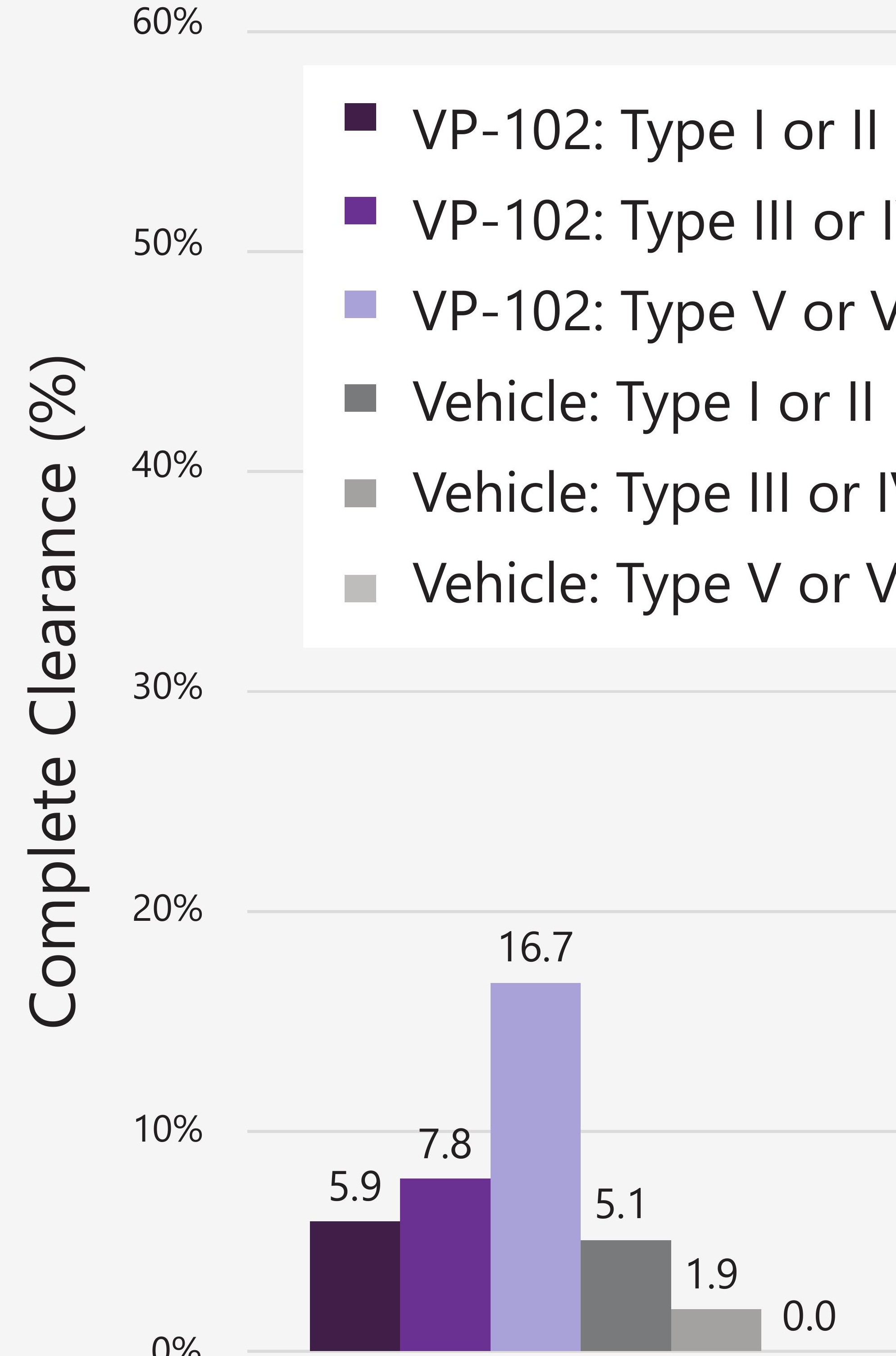

Visit 2 (D)
(Day 21$)$

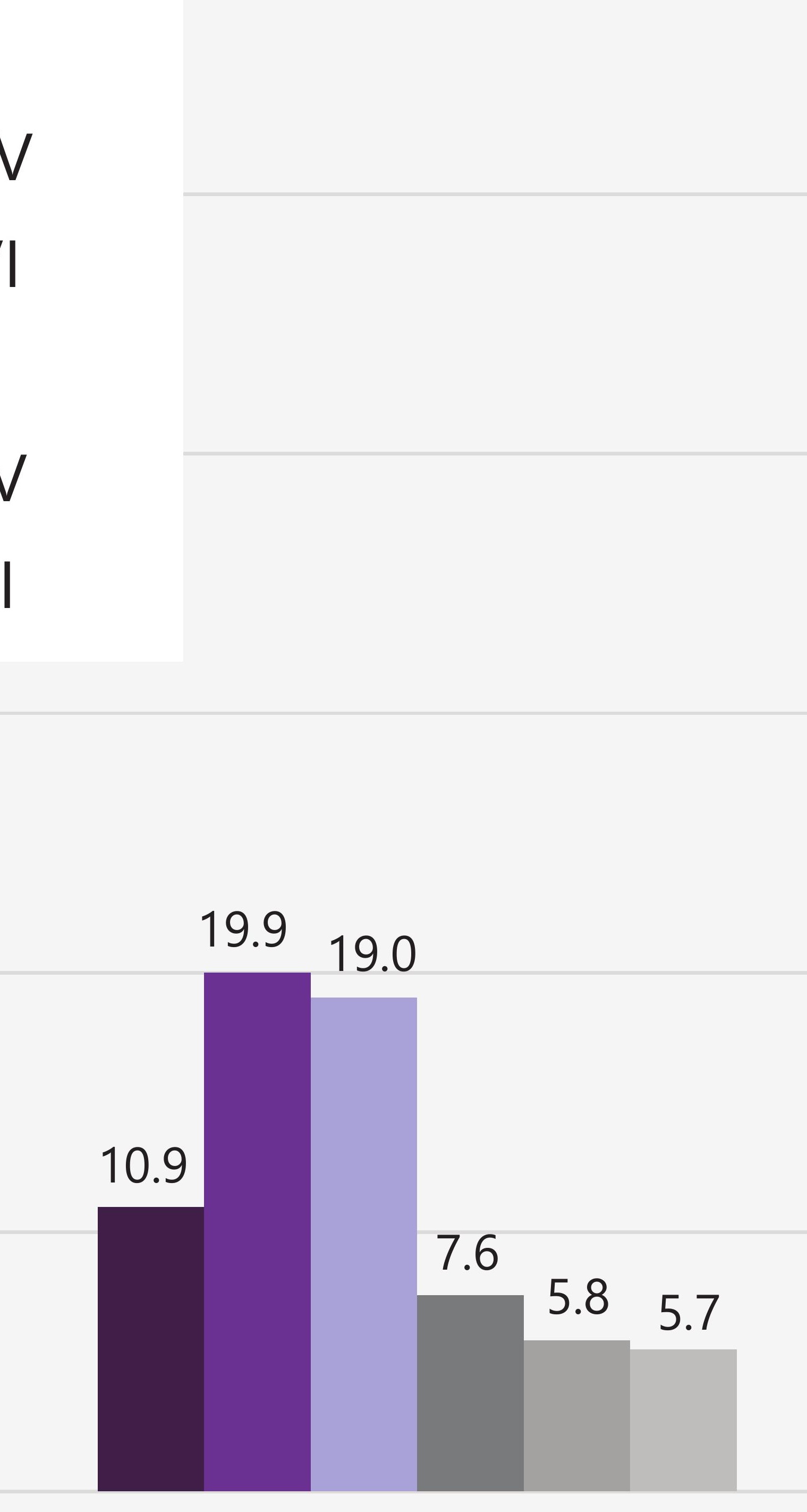

Visit 3
(Day 42)

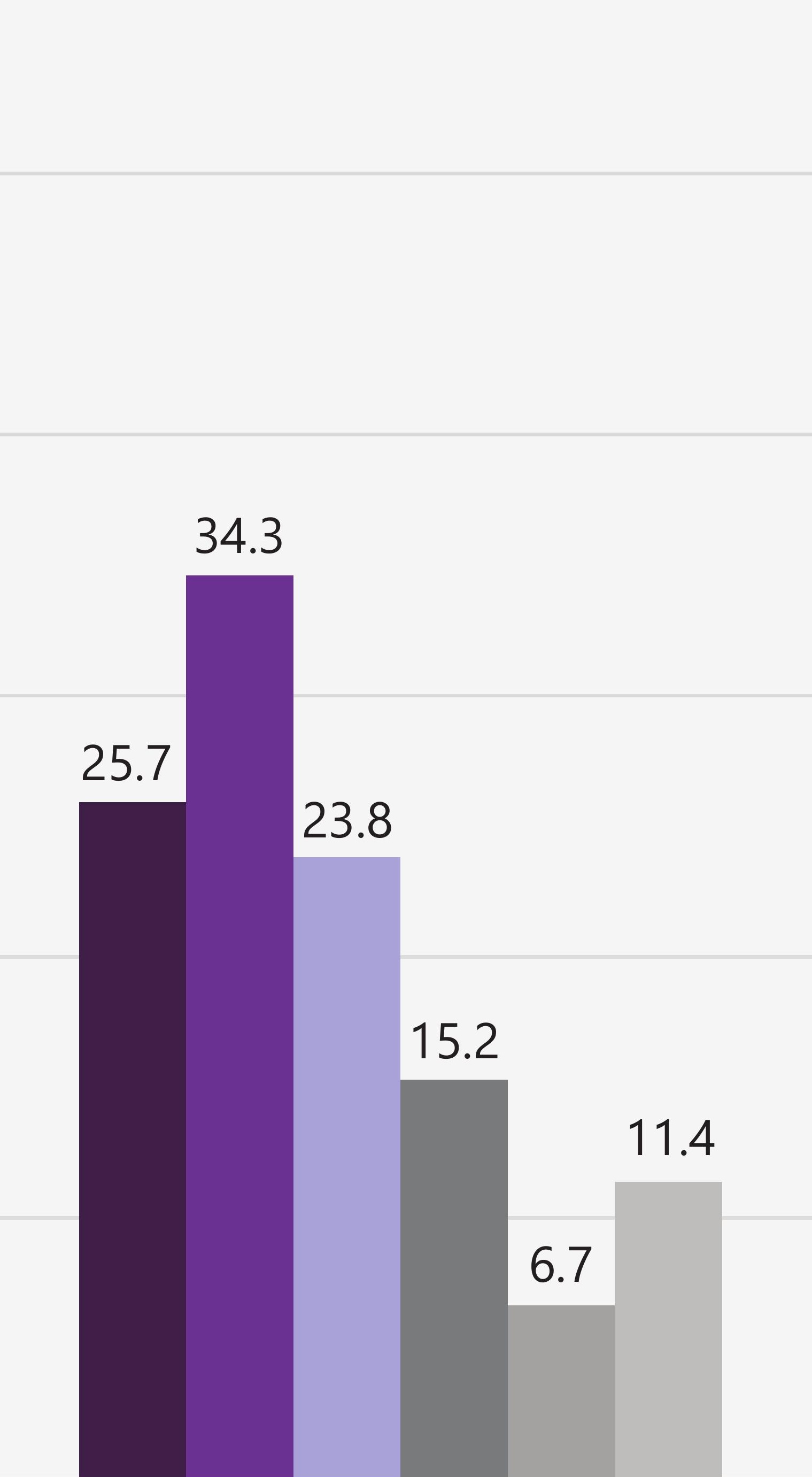

Visit 4
(Day 63)

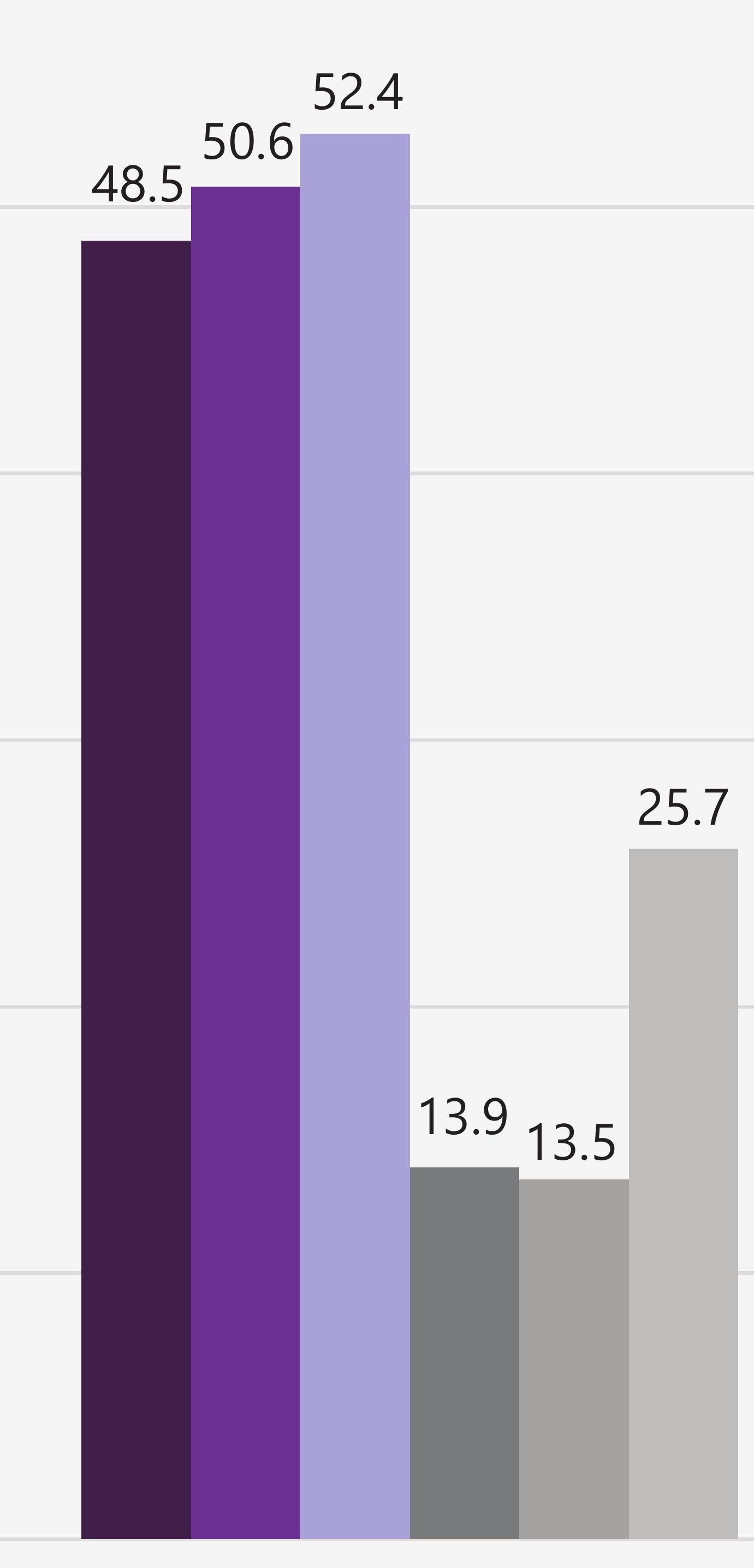

EOS Visit
(Day 84) EOS=end of study

A significantly higher percentage of subjects achieved CC in the VP-102 group than with vehicle at Day 84 VP-102 vs. vehicle ( $50 \%$ vs. $15.6 \%)$ $(p<0.0001)$.

- Mean percent change from baseline in MC lesion counts decreased $76 \%$ for VP-102 and 0.3\% for vehicle at Day $84(p<0.0001)$.

- The most common TEAEs in the VP-102 group were application site blistering, pruritus, pain, and erythema, which were generally mild or moderate in severity. or II ( $n=101$ VP-102; $n=79$ vehicle), 270 with FST III or IV ( $\mathrm{n}=166$ VP-102; $n=104$ vehicle), and 77 with FST V o VI $(n=42$ VP-102; $n=35$ vehicle).

- CC of MC lesions across FST groups was $48.5 \%, 50.6 \%, 52.4 \%$ for types $\mathrm{I} / \mathrm{II}$, III/IV and V/VI when treated with VP-102 and $13.9 \%, 13.5 \%$, and $25.7 \%$ in vehicle group, respectively $p=0.0008$ for type V/VI).

The incidence of TEAEs by FST

subgroup was similar to the overall study population.
Study included 180 subjects with FST I $(p<0.0001$ for types I/III and III/IV, and
CONCLUSION

VP-102 was safe and effective across all Fitzpatrick Skin Type results from the overall study population.

References

1. Ware O et.al. Racial Limitations of Fitzpattick.

2. Eichenfield L et.al. Pooled Results of Two Randomized Phase III Trials
Evaluating VP-102, a Drug-Device Combination Product Containing Treatment of Molluscum Contagiosum 257-265.

Disclosures

The studies were sponsored by Verrica Pharmaceuticals Inc. Editorial support was provided by Versant Learning
Solutions and funded by Verrica Solutions and funded by Verrica Pharmaceutcals lic. The authors Verrica Pharmaceuticals:

E. Siegfried: $H, C ;$ LF Eichenfield: $H, C$ S. P. Kwong: $H, C ;$ S. Desai: consultant; S. Cutler: E; C. Willson: E; P. Rumney.

E; C. Crosby: E; J. Andres: E; M.

McBride: consultant

$\mathrm{H}=$ honoraria; $\mathrm{C}=$ clinical funds: 